\title{
Big Data-based Self Optimization Networking in Multi Carrier Mobile Networks
}

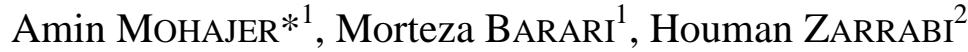 \\ ${ }^{1}$ Faculty of Information, Communications and Security Technology, Malek Ashtar University of \\ Technology, Tehran, Iran \\ ${ }^{2}$ Integrated Network Management Group, ICT Research Institute (ITRC) Tehran, Iran
}

\begin{abstract}
With regard to the increasing growth of demands in order to provide various services and high-speed in new generation of mobile telecommunications, one of the challenges ahead, are the available limited resources. In early cellular systems, with regard to the type of services that were provided, techniques such as the use of this frequency were enough. But in the telecommunications of the next generation, we have to use different techniques and features that are provided with resources in the area covered by the network, leading to an exponential increase in the operational complexity of these kinds of networks will be optimized in the scheme and optimization point of view. Therefore, to achieve an acceptable level of performance on these networks, intercellular performance management will be of great importance. One of the techniques that are recommended to enhance further the efficiency of cellular networks is the usage of self-optimization techniques in these kinds of networks. The foundation of our proposed scheme is presenting a self-optimizing model based on neighboring indices by which we can make feasible the possibility of controlling resources and the respective indices relevant to neighboring connections of cellular network without the interference of human force and merely by relying on the network's intelligence. In order to better the efficiency of the intended scheme, we used the Big Data technique to analyze the data and network's better decision-making process in allocating resources. In a way that in the uplink direction, user's data from the user layer to the network core are analyzed in one register and based on semantic information extracted from these data, the decision-making center will be able to allocate resources in a more intelligent way. The works done on this Category to provide distributed solution of non-self-optimization or has paid for resources allocation that also may not to be close to the optimized solution or have passed up the effect of changes of network conditions. Gained results in the use of this model represent more efficient use of network resources and also reducing the load rate of the imposed signalling on the network.
\end{abstract}

Keywords : Self Organization Networking (SON), Handover, Multi Carrier Mobile Networks, Inter Radio Access Technology (IRAT) 


\section{Introduction}

According to the statistics of $3 \mathrm{GPP}$, the rate of parameters related to the optimization in second generation mobile network to fourth generation networks; it has gained from 500 to about 200 that is actually represents the complexity of such networks. With a glance look to this matter, it can be understood that, practically, optimizing these networks will not possible by manpower. Due to solve this problem, great operators and vendors in the world are towards automatic optimizing of the network. Our purpose in this paper, also, is presenting a self-optimization model with performance of optimizing parameters related to neighboring relations. From the attitude of SSV1, we can, without regard to their effects on the performance of other sites, consider the self-optimization frameworks related to the changes in parameters of the sites. But, in surveying cluster of mobile networks, the neighboring relations and clarifying the levels of accomplishing exchanging of the present sites in a $\mathrm{LA}^{1}$, it has high significance in increasing the efficiency rate of all networks [1]

In continuance and before presenting the suggested model, we will pay attention to reviewing of general concepts that different dimensions of this paper, from various point of view, are in relation with them. These three dimensions are work field, determined purposes and usable instruments. Accordingly, we will have glance to the networks of new mobile generation that are known as our work field in this scheme. Then, self-optimization technology in these kinds of networks will be proposed and technology of big data will be discussed as a tool in making intelligent of network in decision making.

\subsection{The main Challenges}

Stating modern solutions in presenting a self-optimization model in multi-carrier, based on structural characteristics of networks of new generation mobile network can be very useful. But, mentioning this point is necessary that, in order to making realistic of this scheme, we will also face with a lot of challenges. In the continuance, in a case, we will pay attention stating of this kind of challenges.

- The first step in modeling a self-optimization system is finding and collecting suitable information about the complex of user's data [2]. Therefore, it is necessary, by using an automatic system, to find and clarify information related to existences and by a suitable format to enter to system of data analysis. Collecting this information is one of basic challenges in the field of analysis according with big data. So, for collecting

\footnotetext{
${ }^{1}$ Location Area
} 
information related to existing conditions, also it is necessary for a responsible system, to have necessary dynamism.

- Due to completing this scheme and also approaching the scheme to real conditions, it is necessary to use self-optimization scheme that also to have performance ability in the networks of new generation of mobile. On the other hand, according to presented standards in global union of telecommunications, the rate of delay in these networks is very low. Therefore, our presented self-optimization framework should be like a real time. Namely, it is necessary to have a proactive son self-optimization model. And in this case it is necessary to have prediction capability in the self-optimization scheme. With regard to this point that approximately all of posed discussions in past works are around self-optimization is unreal time, it is thought that presenting a different scheme with supporting capability of online services to be along with a lot of problems.

- Being modern of using the methods of data analysis in optimizing mobile networks and entering parameters related to occurrences and without telecommunication statistical parameters along with KPI characteristics, is very important in making intelligence of our self-optimization system. At the moment, this idea is stated only a descriptive idea, and practically we cannot find a scheme, that by using this method, to gain a framework for making more efficient of mobile networks.

In this paper, for obtaining to a valuable and modern, we tried to use the modern tools in new mobile networks. In this regard, telecommunication new discussions are used such as $5 \mathrm{G}$ that will be commercial from 2020 and also technology of new data that is called a big revolution of the era of information technology will be commercial. Combining of these new discussions can, also, be along with problems that in performing this scheme, we will face with them.

\subsection{The Main Goals}

In this paper, by using the methods of big data analysis, our purpose will be obtaining the self-optimization capability in multi carrier networks. After surveying the presented models, it will be tried to present solutions for some present challenges in the new generation mobile networks. Generally, in this research, main focus will be on solving following matters.

- Optimization-based automatic neighboring communications in multi carrier networks in order to getting more efficiency in network

- Presenting a comprehensive and real self-optimization framework in the new generation mobile networks by using big data analysis techniques. 
- Assessing the presented self-optimization model and proving the improvement of its performance in networks with a lot of parameters

- Assurance of efficient performance in the layer model of networks along with multi carriers with suitable relation of microcell and Femtocell layer

Progression towards optimized efficiency in the multi carrier new generation mobile networks is one important and challengeable field in optimizing cellular networks. Up to now, based on for performing optimization process of networks with big criterion, researchers have presented various models. In short, we can introduce some past schemes that have had progressions in this field.

One of the solutions to increase the quality level of the offered service is to reduce the rate of periodical errors during the establishment of connection [4]. Reduction drop rate as well as increasing the rate of successful handover of HOSR can indicate the increase in the quality level of service delivery in cellular networks. In [5], we are dealing with an automatic NCL scheme which has more capabilities compared to similar schemes. In this scheme which is implemented to the core part of the network by adding an administrative register called NMS, the special capability of this scheme in the possibility of network scalability is provided. In [6] the Third-Generation Mobile Networks, the requirements of load distribution in the phase of network design are considered as well [10,11]. The authors in [7] have paid small attention to the degree of transferred power of downlink in addition to the indices which affect the degree of the level of cell's effective coverage.

In addition, in the presented scheme in [8], the authors have introduced the Big Data technologies so as to optimize the fifth-generation of mobile networks which despite the highcapability of this technique in the field of new generation of mobile networks, this scheme has been posed as an idea only and the authors haven't posed the output in order to compare with similar schemes. In other schemes that have been presented in 2014 [9], authors have tried to present a self-optimizing scheme in order to optimize the indices of neighbor relation based on coverage of the available gaps in the covered area of the network which despite the presence of positive and efficient aspects in this scheme, has just considered optimization merely in determining ANR and as the algorithms of this scheme show, this scheme can only be used as a solution to increase the level of successful neighbor relations between the cells $[12,13]$.

As we observed in surveying past schemes, the presented frameworks have defects that generally we can mention them as following:

- Lack of enough notice to the present meaningful information in data 
- The output of many networks intelligent making schemes is usable only in special field and are not usable in other fields.

- Maladjustment of self-optimized models with special conditions of mobile networks specially multi carrier networks of new generation

In the next section, we will pay attention the suggested model. In this model, we have tried to cover the present defects in past schemes.

This paper will be presented as follow: first, after mentioning the basic concepts as introduction part, we indicated to some main previous works which have been proposed before. After that, we present the framework of our scheme in which our idea about providing an efficient self optimization networking model has been introduced. Then we try to examine the result of our scheme through various scenarios in which the effect of our new big data based decision making algorithm has been investigated. Finally we will represent the main references after conclusion part.

\section{Proposed Model}

The general description of our model introduces a framework to overcome the available challenges in the field of NGMN network's optimization by using self-organizing networking SON patters which are based on special features of next generation multi-carrier mobile networks. In this scheme, we introduce a comprehensive framework to reinforce SON with Big Data so that the decision-making algorithm in intelligent optimizing system is promoted and causes an increase in the network efficiency.

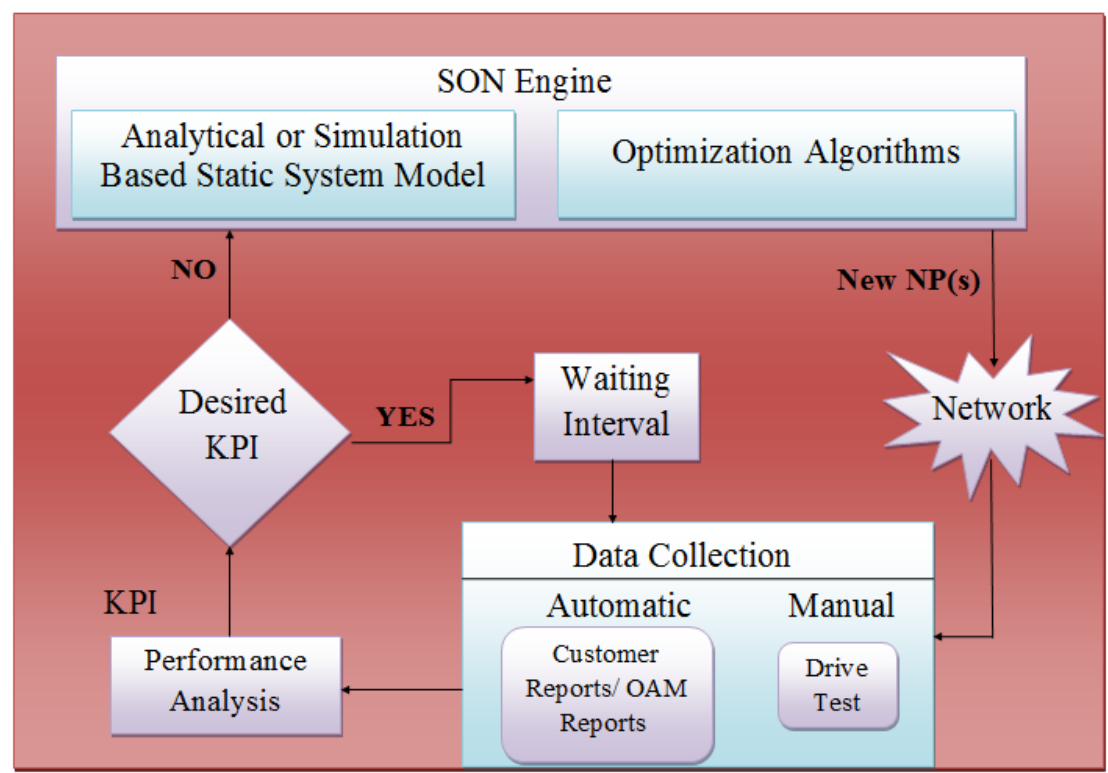

Figure 1: Our methodology in NGMN SON in diagram block. 
Naturally NGMN SON requires abundant intelligence for the emergence of end-to-end networks. A general profile of how to use the self-optimizing motor in controlling efficiency indices are shown in figure 1.

General framework of this scheme has been formed from different parts that have linked like block diagram to each other. These parts can be interpreted in relation with a selforganized system with the technology of big data. But, in continuance, we will pay attention for complete surveying of suggested idea in the framework of this diagram block. It should be noticed that because our work field is next generation mobile networks, so, it is natural that also our way of receiving information will be the way of Up Link of network, from linked things to Femtocells and also different users till the core network [3]. All of received information will be used for analyzing of rate accuracy determination of dedicated sources till we have suitable control on internal and external interference rate. Next, we will survey physical model of Femtocell layer. In this study, we have tried to present an approximate model of optimization relation that has been used in the layer structure of multi carrier network.

The next step in self-optimization networks, by certain approaches that there is measuring management and is able for learning, will be introduced based on past and present observations. This is the method of self-organized networks that are not limited by preintroduced algorithm and in this framework, one part of the network are able to arrange any sudden conditions. In this scheme, we have observed a capability that has been introduced according with this new approach till the nodes of a network also can have the ability of teaching. With performing the algorithms of machine learning, they will be able for exchanging information and teaching each other that will accelerate the process of selfteaching and will cause to faster convergence. Movement towards the changes of fifth generation not only there is in accessible radio network but also there is the section of network core that its new approach makes possible the scheming needed network for presenting services accordance with the users and increasing sets. The trend is like this that separating hardware and software and the movement of functions is one second.

In order to extending the presented model to real conditions, it is necessary to observe the network obligations in different conditions and different periods. As we observed in surveying past works, the affairs that have been posed in the field of optimizing mobile networks and presenting a self-optimization model, it performs the intelligence of network only through analyzing KPI telecommunication efficiency parameters, and they neglect effective non-telecommunication parameters in distributing network traffic and transferring 
network load, and based on, the destructive outcomes that there are in mobile networks due to environmental and social factors, will not be considered in this kind of schemes. In order to implement the plan to self-optimization based on data analysis, we divide the self- parameters into two general following categories. Our purpose is reaching to the self-optimization framework that in this scheme one part of the network, based on supervision on the parameters of these two groups, can predict the conditions of is future and to estimate the rate of its needed sources according network load in every part of the network. The framework that we will present from self-optimization will contain two parts of three parts in performance of self-organized systems.

- Self-optimization

- Self-healing

Three features that distinguish our suggested scheme from common SON technology framework are as following:

- Complete information about the present condition of the network

- Prediction capability of the user's behavior

- Dynamic Capability of network replying to the network parameter modifications

These three capabilities can prepare solutions for scheming the self-organized network that is accordance with the needs of new generations of mobile. Operation of performance blocks in the framework of big data algorithm in the self-optimization scheme is as following stages:

- Collecting data from informational sources into mass data collection, big data

- Massive data conversion to the correct data by developing its plan

- Model: preparing a model of network behavior with learning from accurate data from two-steps

- Performing the engine of self-organizational network: using self-organizational network in model

- Validation: if new $\mathrm{NP}^{2}$ can be prepared by operator's previous knowledge or experience, it will be changed. Otherwise, it simulates the behavior of network for new NPs. If the behavior of stimulation to be according to the observed behavior, new NPs will be accepted.

- Retraining/improvement: if validation to be failed in the fifth stage, the process of changing parameters will be blocked and it will update the behavioral model even if

\footnotetext{
${ }^{2}$ Network Parameter
} 
giving validation have positive outcome, blocking will be occurred periodically to keep the accuracy of the model.

Mentioning this point is necessary that progressed plan in our model is significant from this point of view that by camping a UE on a cell, any more, for creating voice communication with other cells, there is no need to increase the load of signalling, and only that UE is enough to demand for creating conversation to the same cell that simultaneously receives from that PS services. If in that part of network there are neighbors with these features, the best neighbor, for creating communication with best quality according to thresholds will be defined as following:

- Quality Threshold level for applying prioritized Hierarchical cell reselection (QHCS) of neighboring cell when measurement quality is:

- $\mathrm{CPICH}$ EC/NO

- CPICH RSCP

- Quality Offset between serving cell and neighboring cell broadcast

- Timers (such as Penalty time)

That these qualitative assessments are done based on protocols of transferring SIB11 and SIB12 protocols. Meanwhile, care must be taken that the amounts of Inter-System Measurement Trigger threshold for Cell Reselection, determined according to network and environment conditions. In surveying performance in the PS dimension, the presented model continuously will accomplish.

Here care must be taken that handover between systems will be destructive in two aspects. At first stage, IRAT $^{3}$ handover delays will be destructive and in the next stage, when UE takes service from LTE, and we want handover to be accomplished on technology from a lower generation, the quality of given service to UE is decreased strongly. Therefore, handover between systems in the condition of $\mathrm{PS}^{4}$ will have destructive effect rather than $\mathrm{CS}^{5}$. If we arrange the easiest change possible as our first priority, we should notice handover IntraFrequency H.O. in this condition, by surveying the neighboring list, according to two special criteria of the fourth generation we find the best choice: In the process of handover of this kind, some parameters of Cell Reselection are very significant, such as:

Inter-System Measurement Trigger Threshold for Cell Reselection

\footnotetext{
${ }^{3}$ Inter Radio Access Technology (IRAT)

${ }^{4}$ Packet Services

${ }^{5}$ Circuit Switch
} 
The function of using the Big Data technique in specifying the threshold limit of availability in the presented self-optimizing model is that we can prevent the over-needed increase in network signalling load by logical allocation of resources to different parts of the network in different times as well as overusing the network resources such as the power. Situational conditions as well as the popularity distribution of the subscribers of an operator varied during a time slot hence the use level of network resources must be varied during this time slot. This case is either not considered in the current cellular telecommunication or if it is, very little attention is being paid to it. In order to use all these information in setting the network parameters, we have considered telecommunication systemic factors along with other effective factors in 3 groups which the classification of the set of these parameters and factors can be shown as the following.

- Network Conditional Variables

- Non-telecom parameters

- The calendar index

- The movement direction of the subscribers

- The index of traffic distribution in a day (The day and night phenomenon)

- Atmospheric effects

The analysis that has been done on these factors, direct us toward the precise selection of threshold limitation used in functional algorithms of the self-optimizing model. Generally, the problem can be stated in this way that our self-optimizing model has the capability to predict the situational condition and the network status and to ever optimize the network performance, this model adjusts the network in a way that the extra resources don't get used in the network. Also in emergency situations in which those extra resources are needed, the network's mechanism changes in a way that the quality of communication is maintained.

In order to implement this idea in the self-optimizing model, we group the previously mentioned factors along with their effectiveness coefficients into a relationship and the obtained answer amount will indicate network status in terms of those parameters. In order to using the fuzzy logic concept through our decision making algorithm, we should classify all of effective parameters and calculate the result based on the coefficient related to each parameter. In our scheme these parameters are Day \& Night, calendar which indicates the social events, population distribution and so on. Finally the extracted result from decision algorithm which indicates the status of the network, should compare with the predefined threshold. 
Our self-optimization model will have the capability of estimating each of these conditions, and also the threshold will be arranged according to the condition that there is network in it. But in order to make more precise decision, we use the method of fuzzy parameters. In this case, instead of seeing variables as binary perspective, we also consider the middle degree of moods. In this case, every effective variable in its decision making is assessed in three or more moods.

According to the figure 2, by surveying certain threshold for every variable, the mood of that variable will be determined in a period of time.

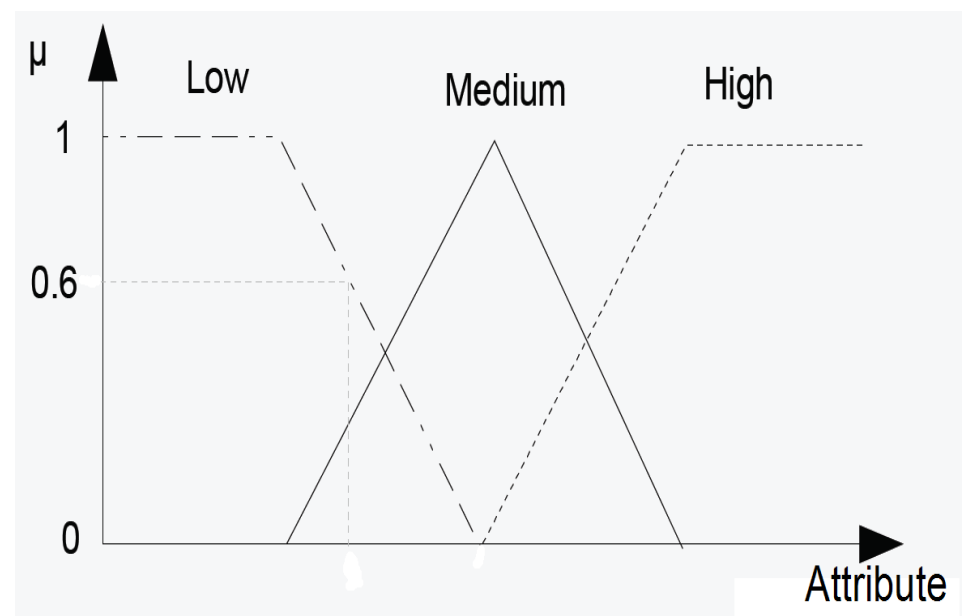

Figure 2: Grading the effective variable in the process of handover.

Based on the rate of effectiveness of each criteria on decision making, a coefficient is devoted to every of these variables. In these conditions, with having the level of variables and by specifying the coefficient of their effectiveness, the output is a digit that will be our base of decision making for doing handover, as it is shown in figure 3. As an example, if $D \& N^{6}$ to be in the condition of Busy Hour, $\mathrm{UWC}^{7}$ to have normal condition $\mathrm{TEP}^{8}$ to be in High condition, in these conditions we will have:

$\underline{\boldsymbol{D} \boldsymbol{N}}=\{$ Day, Night, Busy Hour $\}$

$\underline{\boldsymbol{U W C}}$ : Unstable Weather Condition $=\{$ Low, Normal, High $\}$

$\underline{\boldsymbol{T P E}}:$ Temporal Populated Event $=\{$ Low, Normal, High $\}$

$\underline{\text { Handover Factor }}=$ OUT $\{(\mathrm{D} \& N$, UWC, TPE $)$, Telecom Parameters $\}$

\footnotetext{
${ }^{6}$ Day \& Night

${ }^{7}$ Unstable Weather Condition

${ }^{8}$ Temporal Populated Event
} 
Bulletin de la Société Royale des Sciences de Liège, Vol. 85, 2016, p. 392 - 408

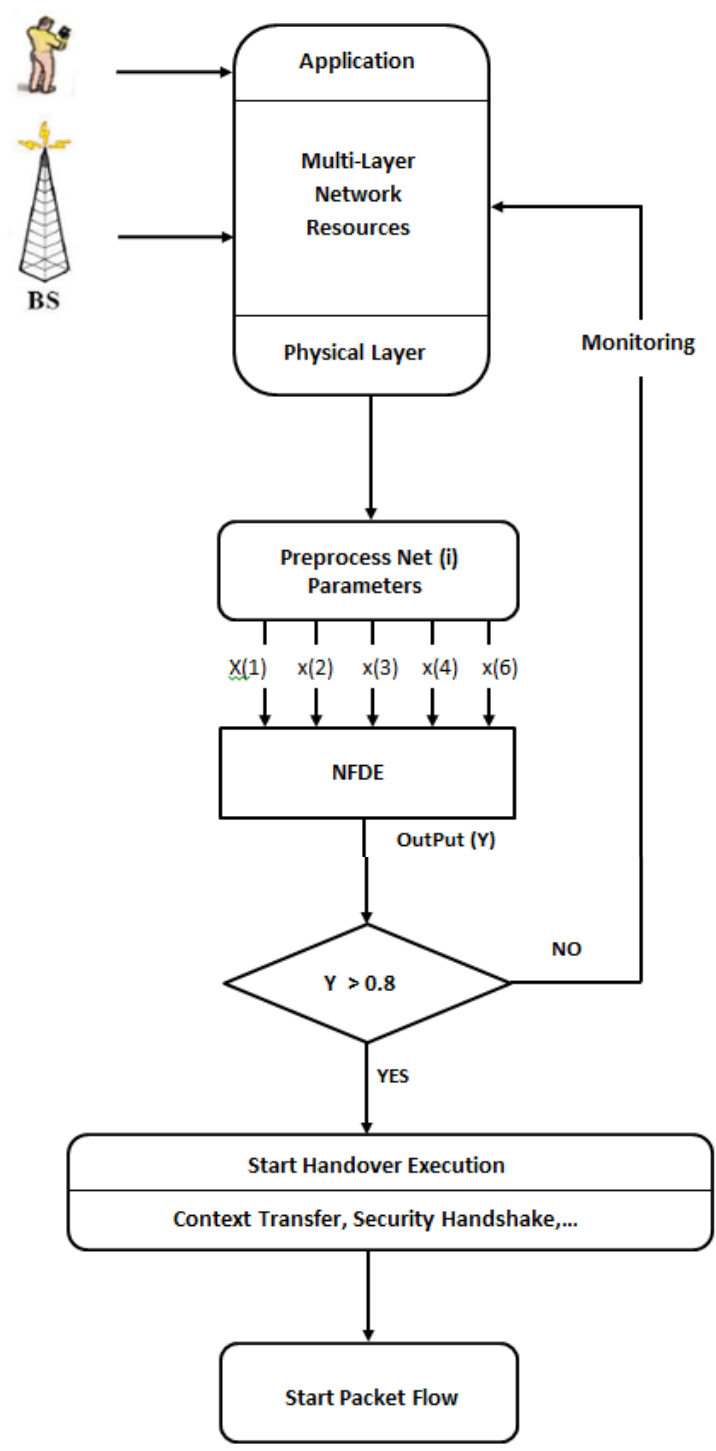

Figure 3: Multiple Decision making algorithm

So, by this approach, we can take big steps in using the technology of big data in moving towards optimizing next generation mobile networks. Naturally, by completing the presented table and also by adding on the number of effective parameters on network efficiency, we can optimize this plan more than before. In next step, we will try to develop the dimensions of this model both in increasing capabilities of applied algorithms and also completing the analysis of effective parameters on the condition of the network. 


\section{Experimental Results: KPI Evaluation}

In order to assess the results of suggested plan, we will try to analyze the output of performing suggested model in a cluster with five base station and 15 cells in KPI mode. Due to a lot of population concentration in two half of the year, this cluster can, as a suitable field for surveying the output of suggested model, be considered. By surveying KPI in primary half that the concentration of population is high, there has been observed this network from the attitude rate of sources and efficiency is in a suitable condition. Now, in the second half that the rate of population concentration is decreasing, if the sources of network are used like past, the rate of the efficiency of the network will be criticized ${ }^{9}$. In these conditions, the presented optimized model, in order to devoting signalling sources to the traffic sources and increasing rate of transferring data, will have an approach based on decreasing the rate of signalling load. Due to this reason, by decreasing handover attempt rate, practically the rate of frequency interferences have been decreased and the quality of channels will increase, and anymore there is no need to create more signalling for creating new neighboring relations. For this reason, by hardening the threshold of handover, we possibly decrease the rate of signalling relations related to transferring service from one site to another site.

Mentioning this point is necessary that the number of users to be high, there are need for so many handover in the network; because the traffic load of network must be arranged based on the rate of present sources in every point. But in a condition that the number of users to be down, every cell can, in most cases, prepare its needed sources under its zone, except fluid users that in most cases of servicing to them, is along with handovers.

As it is observed in figure 4, with increasing the threshold of decision making for handover, the rate of handover attempt will be decreased noticeably. In continuance of effects, this change will be surveyed in the levels of other parameters KPI.

\footnotetext{
9 In order to better understanding the existing relations in the cluster based cellular channels, the sources of 3 GPP can be useful.
} 


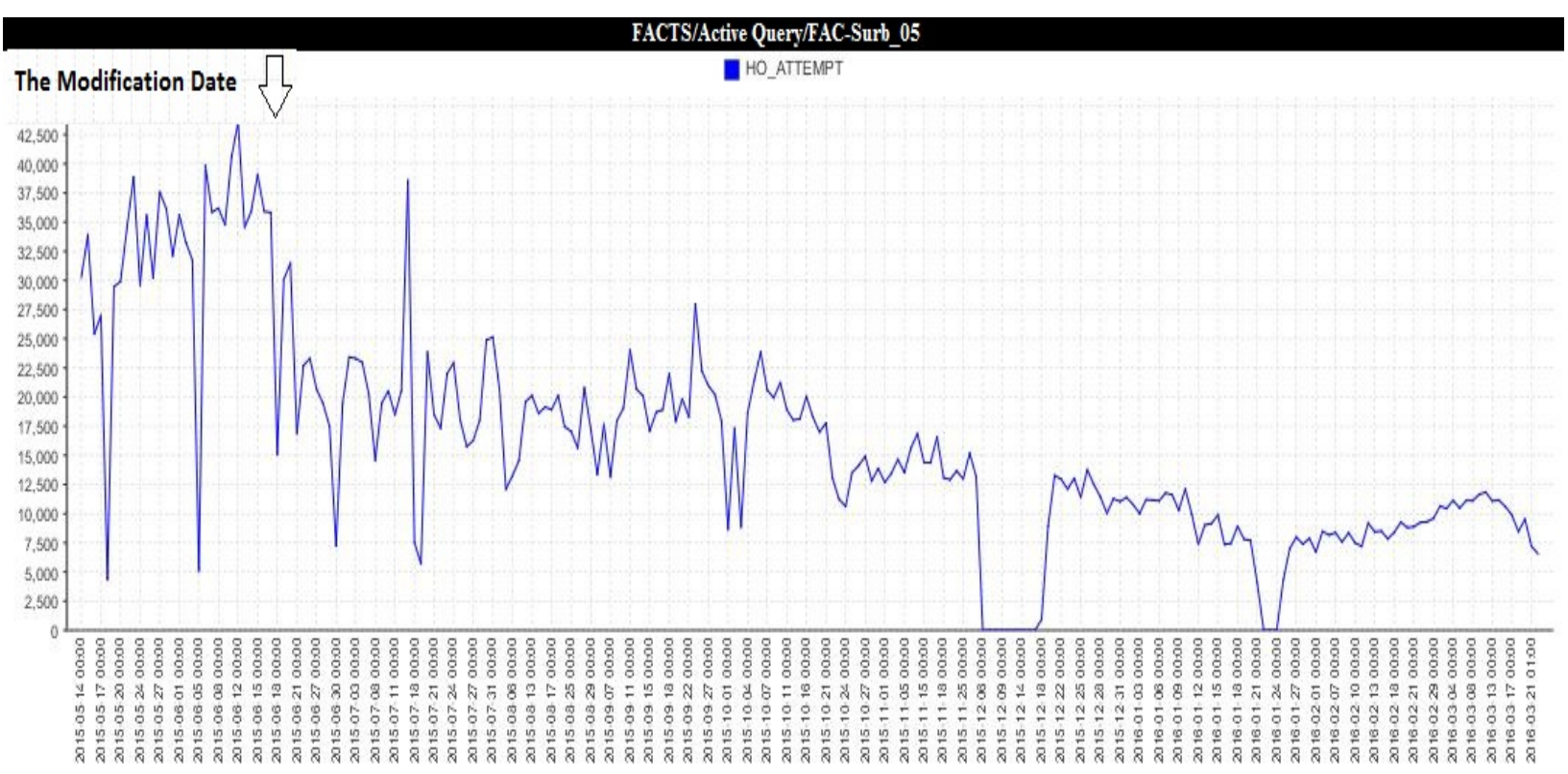

Figure 4: Decreasing the rate of handover attempt in period of assessing cluster, before and after doing changes

With regard to this point that the number of demands have been decreased noticeably for handover, but, successful rate of handover (HOSR) only has been decreased a few percent. As it is observed in figure 5, this rate of reduction, will not have negative effect on the efficiency of the network.

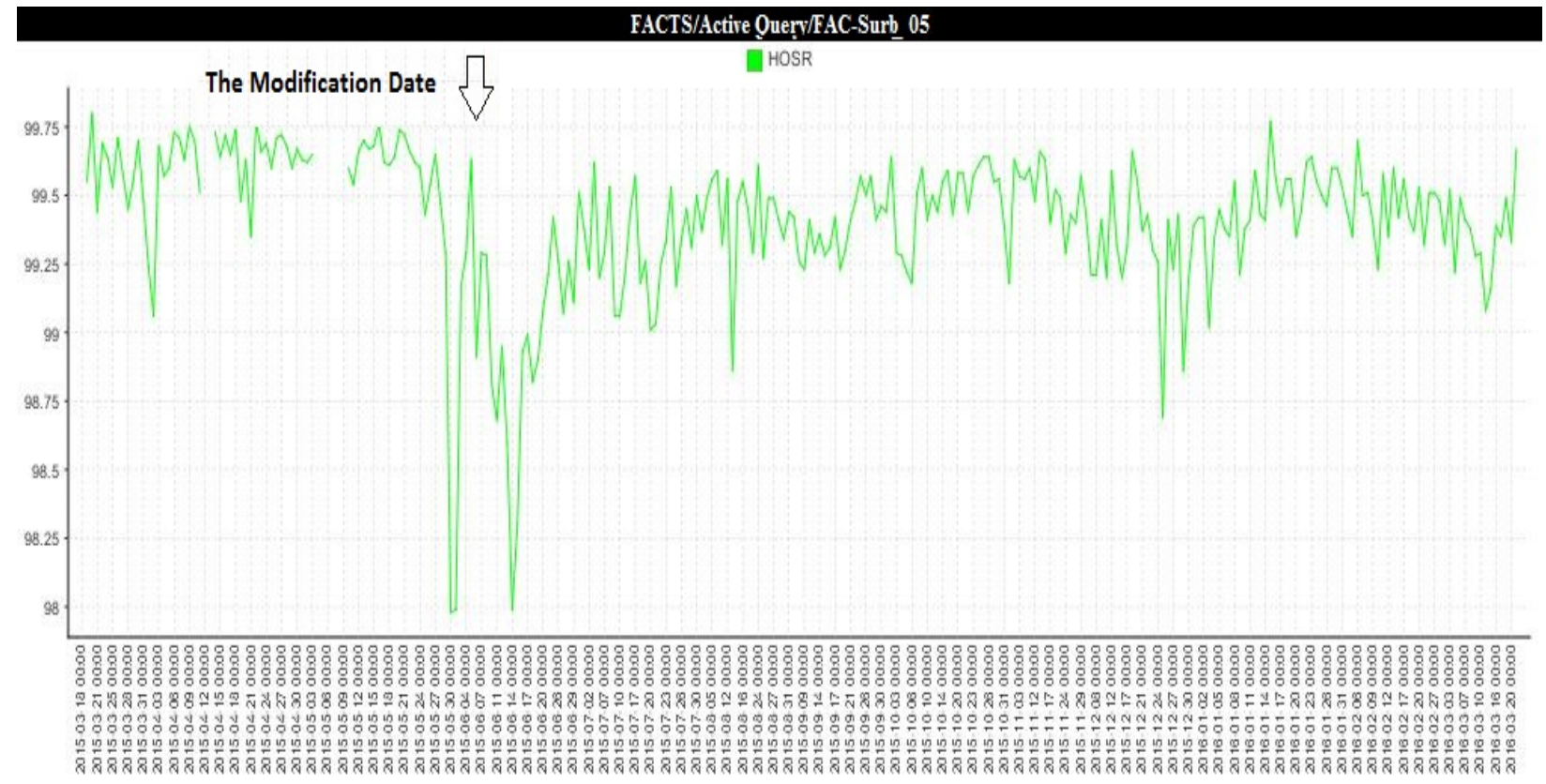

Figure 5: The handover success rate before and after doing modifications

And but, main effectiveness of hardening conditions of handover is in reducing the load of signalling in the network. It is clear in figure 6 that after doing this changing in network, the load of signalling has had reduction $\% 30$ that this case, for getting to an optimized network 
can be very significant. With regard to these conditions, we can devote unusable sources of network signalling to traffic channels for transferring data.

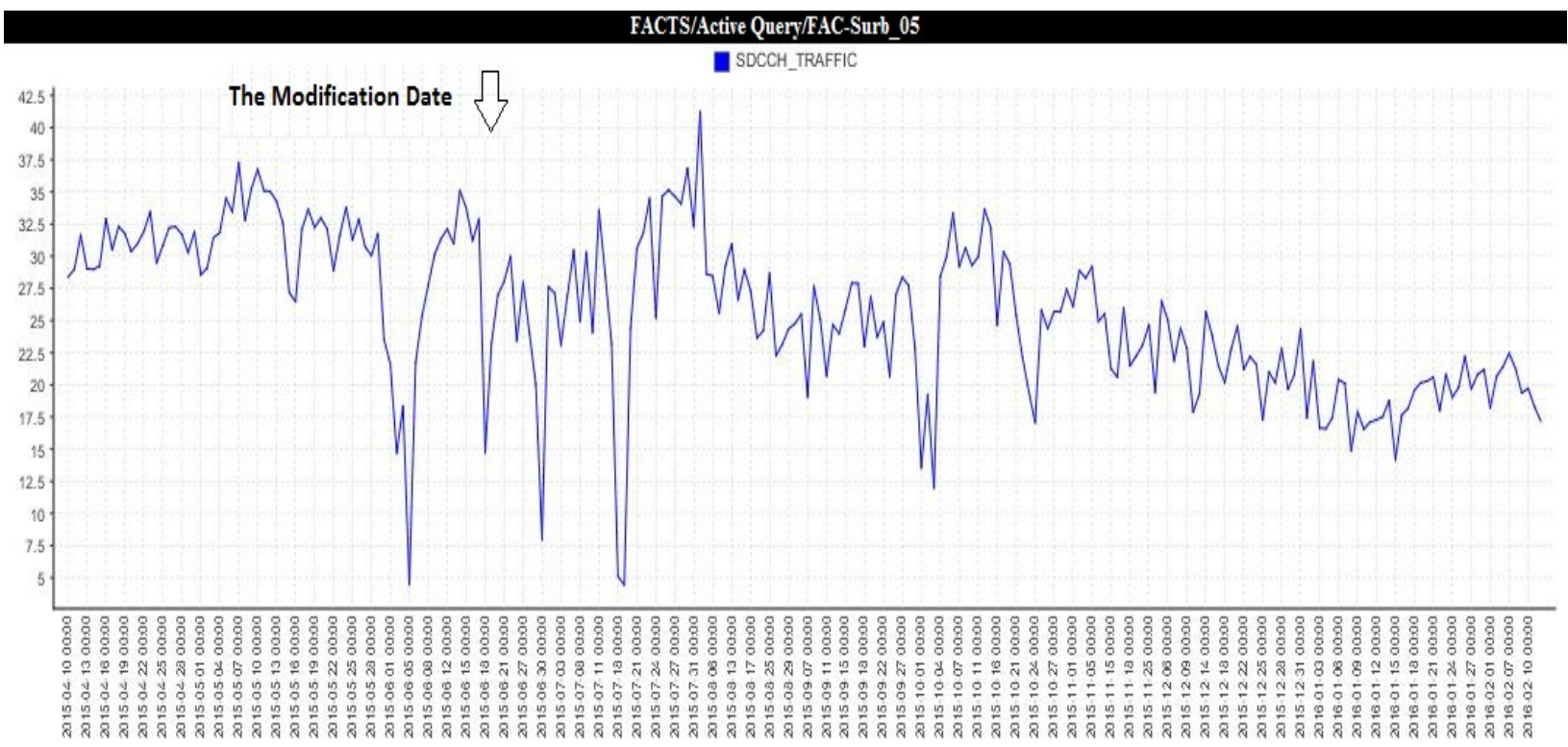

Figure 6: 30 percent reduction of average rate of traffic load in signalling channels for every site of cluster

One of the cases that should be taken into account is this point that by reducing control signals changes whether the conditions of network retainability preserving index and the quality of retain ability or not.

As indicated in figure 7, negative change in the rate of quality in uplink and downlinks is not observable that of course this lack of reducing quality is also related to the reduction of receiver service.

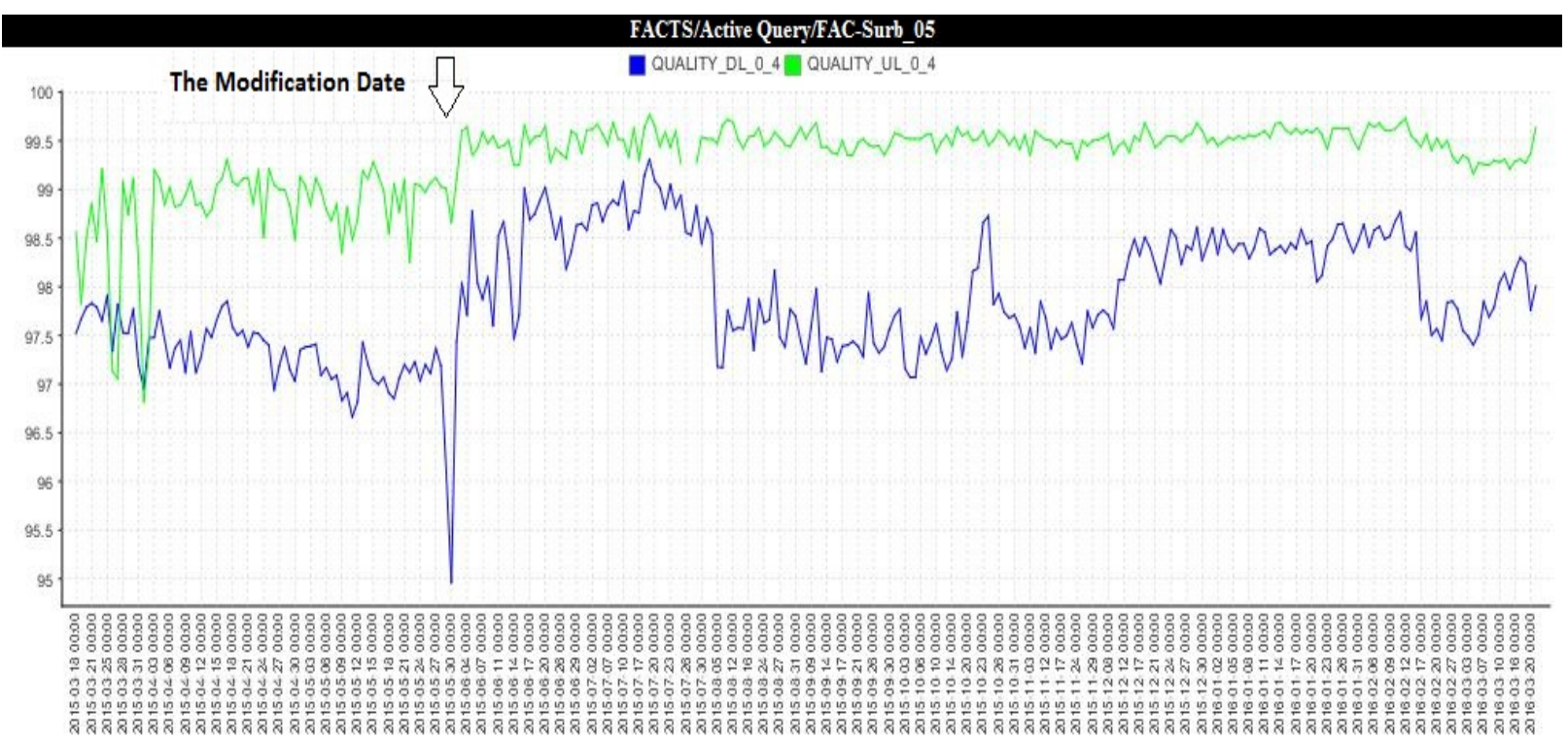

Figure 7: Signal quality level in uplink and downlink 
By reducing the rate of signalling sources we should not face with blocking in demand of control channel. Surveying in figure 8 indicate that the rate of blocking of control channels are without change, and after doing changes in the network, there is not an observable case of concentration. But the rate of loss and drop call, partly after limiting the condition of handover, is clear that this rate is very inconsiderable. The reason of less increase of the rate of call drop is due to this reason that by hardening the threshold constraints, practically majority of services in every zone is prepared by cellular sources of the same zone, and due to being island of this cluster with being far of service-receiver users from the limitation of cluster, little cells before can undertake the function of user service.

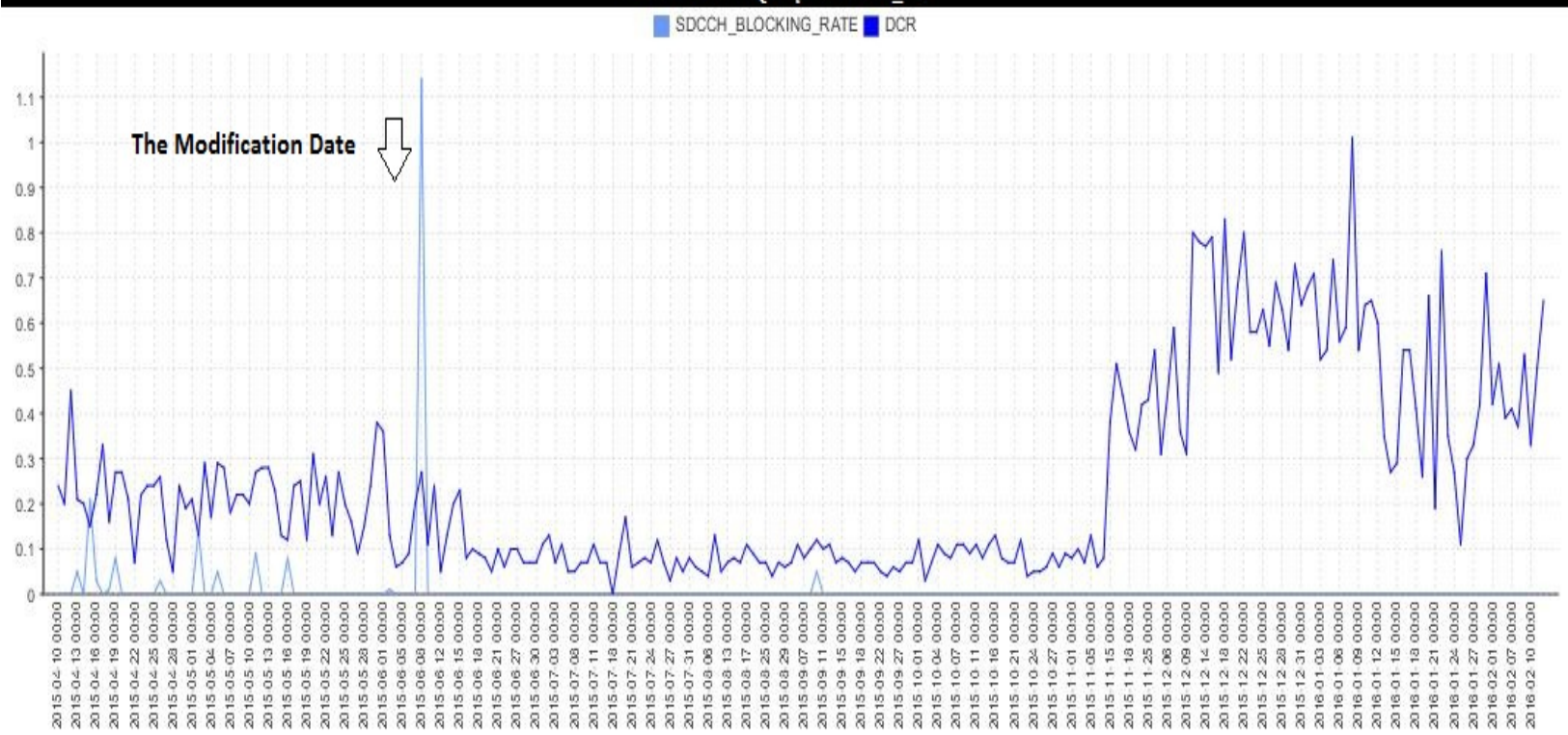

Figure 8: Rate of blocking in signalling channels/ Call Drop Rate

And but, as it is visible in the figure 9, by devoting radio sources to the traffic channels of data transferring, rate of transferring data have been increased significantly specially in downlink. Of course, decrease of the number of service-receiver users also is involved in this matter. But, correspondence of this increase of rate is completely obvious with the rate of traffic sources. 


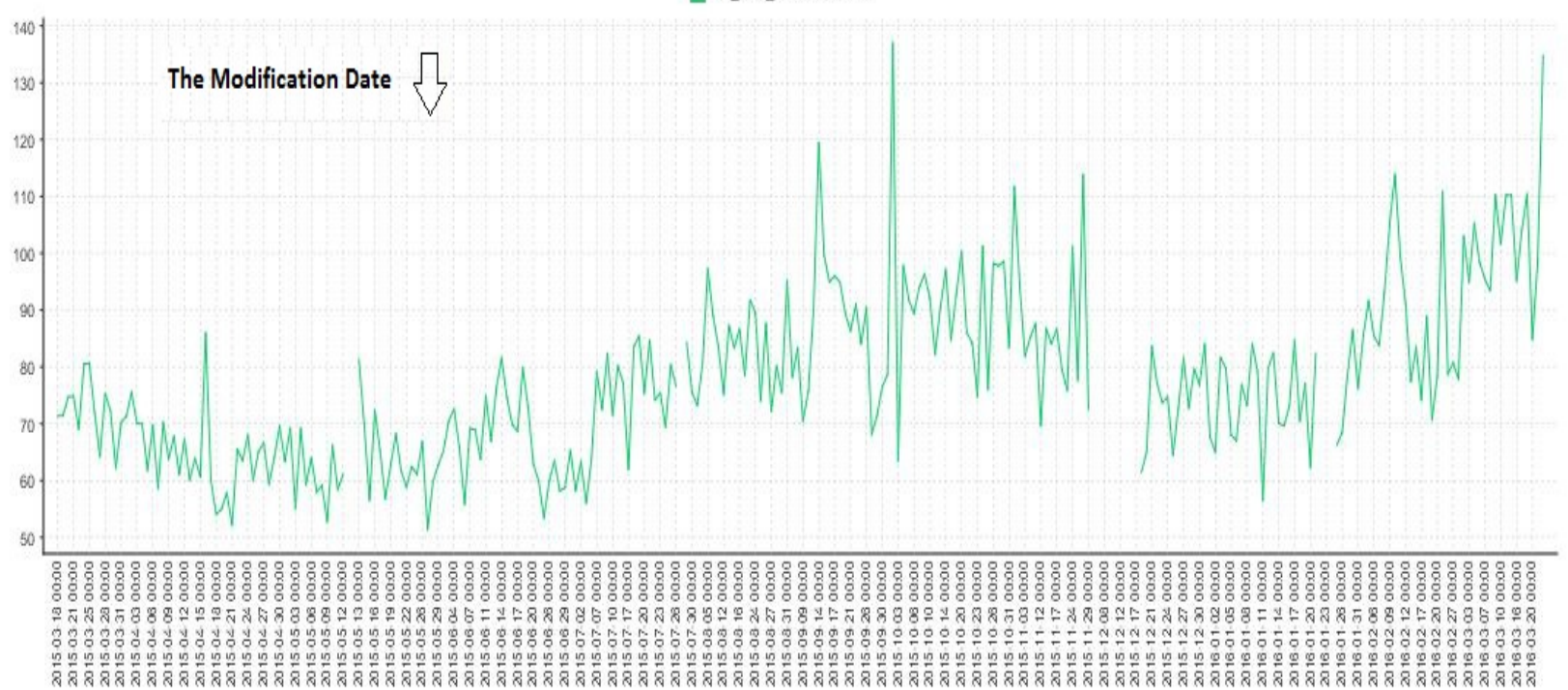

Figure 9: Increasing the rate of sending data after devoting signalling sources to the traffic channels

\section{Conclusion and Future Works}

While self-organizing network model was developed during the past few decades so that automatizes second and third and fourth generation of mobile communications, but due to the method of its reactive scheming and lack of end-to-end network, framework that is schemed is not in accordance with the requirements of the fifth generation. To investigate these problems, strengthening self-organizing network with data analysis tools such as technology of Big Data is posed that in using this technology, with direct intervention in the transmitted data to achieve the concepts, by grouping models of transferring data and by providing services, to create new performance models.

As it was seen in this paper, our activity toward presenting a self-optimizing model based on Big Data was done in two aspects which one dimension of it was related to presenting a selfoptimizing model for handover communications on an inter-cellular communication level which by making this model smart, the possibility to access the maximum efficiency of the network in different environmental conditions and popularity distribution won't be out of reach. In addition, in the second dimension of the scheme, implementing different procedures in the network's functional mechanism is analyzed by using the available data assessment in current mobile networks and the practicality horizon of the proposed self-optimizing model can be considerably widened by analyzing the obtained outputs. Also, from amongst the most important works that can be done as follow-up studies to the one done in this paper is 
presenting an empirical model between the parameters already introduced that naturally for an ever better description of this model, large amount of data must be evaluated.

\section{References}

[1] 3GPP TS 36.413 (September 2011) Evolved Universal Terrestrial Radio Access Network (E-UTRAN); S1 Application Protocol (S1AP), Release 10, section 8.7.3.

[2] M. Matti and T. Kvernvik, "Applying big-data technologies to ntwork architecture," Ericsson Review, 2012.

[3] M. Feng, D. Chen, Z. Wang, T. Jiang, “Throughput improvement for OFDMA femtocell networks through spectrum allocation and access control strategy," IEEE Computing, Communications and applications Conferencce (ComComAp), pp. 387-391, 2012.

[4] NGMN, "NGMN Recommendation on SON and O\&M Requirements," Dec. 2008.

[5] 3GPP, "Self-configuring and self-optimizing network use cases and solutions," Technical Report TR36.902, http://www.3gpp.org/

[6] S. Magnusson and H. Olofsson, "Dynamic Neighbor Cell List Planning in a Micro Cellular Network," IEEE International Conference on Universal Personal Communications, San Diego, CA, USA, 1997.

[7] D. Soldani and I. Ore, "Self-Optimizing Neighbor Cell Lists for UTRA FDD Networks Using Detected Set Reporting," IEEE Vehicular Technology Conference, 2007.

[8] A. Imran and A.Zoha, "Challenges in 5G: How to Empower SON with Big Data for Enabling 5G” IEEE Network Journal. November/December 2014

[9] C. L. Lee, W. S. Su, K. Tang, "Scheme of handover self-optimization using big data analytics

[10] P. K. Frenger, P. Orten, "Code-spread CDMA with interference cancellation," IEEE Journal on Selected Areas in Communications, vol. 17, no. 12, pp. 2090-2095, 1999.

[11] A. Duel-Hallen, J. Holtzman, "Multiuser detection for CDMA systems, "IEEE Personal Communications, vol. 2, pp. 46-58, 1995.

[12] P. Xia, V. Chandrasekhar, "Openn vs. closed access femtocells in the uplink, "IEEE Transactions on Wireless communications, vol. 9, no. 12, pp. 3798-3809, 2010.

[13] V. Chandrasekhar, J. G. Andrews, "Distributed power control in femtocell-underlay cellular networks" IEEE Global Telecommunications Conference, GLOBECOM 2009., pp. 16, 2009. 